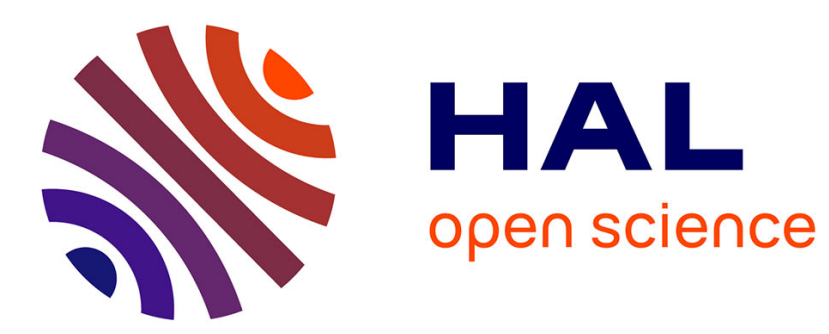

\title{
Modeling and performance of uncoated microcantilever-based chemical sensors
}

Sébastien Tétin, Benjamin Caillard, Francis Ménil, Hélène Debéda, Claude Lucat, Claude Pellet, Isabelle Dufour

\section{- To cite this version:}

Sébastien Tétin, Benjamin Caillard, Francis Ménil, Hélène Debéda, Claude Lucat, et al.. Modeling and performance of uncoated microcantilever-based chemical sensors. Sensors and Actuators B: Chemical, 2010, 143, pp.555-560. 10.1016/j.snb.2009.09.062 . hal-00453482

HAL Id: hal-00453482

https://hal.science/hal-00453482

Submitted on 13 Nov 2014

HAL is a multi-disciplinary open access archive for the deposit and dissemination of scientific research documents, whether they are published or not. The documents may come from teaching and research institutions in France or abroad, or from public or private research centers.
L'archive ouverte pluridisciplinaire HAL, est destinée au dépôt et à la diffusion de documents scientifiques de niveau recherche, publiés ou non, émanant des établissements d'enseignement et de recherche français ou étrangers, des laboratoires publics ou privés. 
Preprint - Sensors and Actuators B, 143, 2010, pp. 555-560. (doi: 10.1016/j.snb.2009.09.062).

\title{
Modeling and Performance of Uncoated Microcantilever-based Chemical Sensors
}

Sébastien Tétin, Benjamin Caillard, Francis Ménil, Hélène Debéda, Claude Lucat, Claude Pellet, Isabelle Dufour

Université de Bordeaux

IMS Laboratory

33405 Talence, France

Corresponding author: Isabelle Dufour

isabelle.dufour@ims-bordeaux.fr

tel: +33540006554

fax: +33556371545

\begin{abstract}
Chemical sensors based on vibrating silicon microcantilevers without sensitive coating are investigated herein. The sensor signal is the relative variation of the microcantilever resonant frequency which depends on both the viscosity and the density of the fluid surrounding the microcantilever. This principle has been applied to the detection of binary gas mixtures. Experimental data for $\mathrm{He} / \mathrm{N}_{2}$ and $\mathrm{CO}_{2} / \mathrm{N}_{2}$ environments are presented and compared to results of theoretical modeling. The advantages of such a gas sensor based on changes of physical properties are discussed (response time, sensitivity, selectivity, stability).
\end{abstract}


Preprint - Sensors and Actuators B, 143, 2010, pp. 555-560. (doi: 10.1016/j.snb.2009.09.062).

\section{INTRODUCTION}

During the last decade, the use of resonant microcantilevers has involved a significant advance in the field of chemical sensors $[1,2]$. For this type of sensor the standard use is based on depositing a sensitive coating (usually a polymer or inorganic porous material) on a silicon microcantilever. Then, the specific sorption of the target gas by the sensitive coating modifies the mass of the microstructure and consequently its resonant frequency $[1,2]$.

An alternative way to use a vibrating silicon microcantilever, without a sensitive coating, as a chemical sensor is proposed here. Using the fact that the resonant frequency of a microcantilever depends on the density and viscosity of the surrounding medium, it is possible to determine the concentration of a gas in a binary mixture.

In the first part of this paper, the analytical expression of the resonant frequency shift due to a modification of either the density or the viscosity of the fluid is presented. Then examples of measurements in binary gas mixtures $\left(\mathrm{He} / \mathrm{N}_{2}\right.$ and $\left.\mathrm{CO}_{2} / \mathrm{N}_{2}\right)$ are shown. In the last part, the measurements are compared to the analytical modeling results and the performance of such chemical microsensors is examined and compared to that of classical coated microcantilever-based chemical sensors.

\section{ANALYTICAL EXPRESSION FOR THE FREQUENCY SHIFT}

When a microcantilever vibrates in a viscous fluid (gas or liquid), the fluid offers resistance to the motion. The fluid loading on the beam includes an inertial force, proportional to the beam acceleration, and a viscous or dissipative force that is proportional to the microcantilever velocity [3-5]. These fluid effects influence the dynamic response of the beam, in particular, the resonant frequency $f_{r}[5]$ :

$$
f_{r}=f_{0} \frac{1}{\sqrt{1+L g_{2} / m}} \sqrt{1-\frac{1}{2 Q^{2}}}
$$

Here $f_{0}$ is the undamped natural frequency of the microcantilever in vacuum, $Q$ is the quality factor of the cantilever/fluid system (depending on the losses), $g_{2}$ is a fluid-dependent inertia parameter (defined later), and $m$ is the microcantilever mass. 
Preprint - Sensors and Actuators B, 143, 2010, pp. 555-560. (doi: 10.1016/j.snb.2009.09.062).

The classical result for the natural frequency $f_{0}$ in vacuum is [6]

$$
f_{0}=\frac{\lambda_{0}^{2}}{2 \pi L^{2}} \sqrt{\frac{E I L}{m}}
$$

with $\lambda_{0}=1.875$ corresponding to the fundamental flexural mode, $L$ the cantilever length, $E$ the Young modulus and $I$ the moment of inertia of the microcantilever cross section about the axis of bending, given by $I=b h^{3} / 12$, with $b$ and $h$ the width and the thickness of the microcantilever.

The quality factor $Q$ of a microcantilever sensor in a fluid environment depends on the system energy losses. In general, these losses may be intrinsic (thermoelastic losses, clamping losses, bulk internal friction other than thermoelastic dissipation, etc.) $[7,8]$ or due to the surrounding medium (viscous losses). In the case of gas chemical sensors, the surrounding medium is typically a gas at atmospheric pressure. In our case (no coating), the losses due to viscous damping in the fluid are dominant [3], and the results of Sader [5] are applicable. Using the current notation, Sader's expression for the quality factor $Q$ may be written as

$$
Q=\frac{2 \pi \sqrt{1+L g_{2} / m}}{L g_{1} / m} f_{0}
$$

where $g_{1}$ and $g_{2}$ are the fluid-dependent viscosity and inertia parameters, given explicitly by:

$$
\begin{gathered}
g_{1}=\frac{\pi^{2} \rho_{f} b^{2} f_{r}}{2} \Gamma_{i}\left(f_{r}\right) \\
g_{2}=\frac{\pi \rho_{f} b^{2}}{4} \Gamma_{r}\left(f_{r}\right)
\end{gathered}
$$

where $\rho_{f}$ is the fluid mass density, $\eta$ is the fluid viscosity, and $\Gamma_{\mathrm{r}}$ and $\Gamma_{\mathrm{i}}$ are the real and imaginary parts of the (dimensionless) "hydrodynamic function": 
Preprint - Sensors and Actuators B, 143, 2010, pp. 555-560. (doi: 10.1016/j.snb.2009.09.062).

$$
\begin{gathered}
\Gamma_{\mathrm{r}}=a_{1}+a_{2} \frac{\delta}{b} \\
\Gamma_{\mathrm{i}}=b_{1} \frac{\delta}{b}+b_{2}\left(\frac{\delta}{b}\right)^{2}
\end{gathered}
$$

with $a_{1}=1.0553, a_{2}=3.7997, b_{1}=3.8018$ and $b_{2}=2.7364$ being Maali's parameters [9] and $\delta$ the length representing the thickness of the thin viscous layer surrounding the microcantilever in which the velocity has dropped by a factor of $1 / e=0.37$, which can be expressed by

$$
\delta=\sqrt{\frac{\eta}{\pi \rho_{f} f_{r}}}
$$

Considering small variations of fluid density and fluid viscosity it is possible to obtain the analytical expression of the resonant frequency shift due to these fluid property variations. Using equations 1-8, the resonant frequency shift can be approximated with a first-order approximation:

$$
\frac{d f_{r}}{f_{r}}=-\frac{\pi}{8} \frac{\rho_{f}}{\rho} \frac{1}{h}\left(a_{1} b \frac{d \rho_{f}}{\rho_{f}}+\frac{a_{2} \delta}{2} \frac{d \eta}{\eta}\right)
$$

where $\rho$ is the density of the microcantilever. This analytical expression relates the relative resonant frequency shift to the mass density and viscosity of the fluid and also to the microcantilever geometrical parameters.

Remarks concerning the assumptions:

All the previous equations (1-9) are considered valid provided that [5]:

- The amplitude of vibration is much smaller than the microcantilever thickness; thus, there is no nonlinear term in the differential equation governing the microcantilever vibration.

- The microcantilever length is sufficiently larger than both its width and its thickness. In this case the equation for the microcantilever vibration is a one-dimensional beam equation. 
Preprint - Sensors and Actuators B, 143, 2010, pp. 555-560. (doi: 10.1016/j.snb.2009.09.062).

- The fluid can be assumed to be incompressible. For this, the wavelength of vibration must exceed both the dominant length scale in the flow, $b$, and the amplitude of vibration. Moreover, in a gas phase medium, the assumption of incompressibility should be supplemented by the condition that the dominant length scale in the flow, $b$, is sufficiently greater than the mean free path of the gas.

In this paper, all of these assumptions are verified. In fact, the microcantilever geometry satisfies $L>b$ and $L>>h$. Moreover, all the gases are used at atmospheric pressure and the microcantilever geometry is in the micrometer range consequently gases can be considered incompressible fluids. Different groups [9-10] have already verified experimentally the equations 1-8 in the case of vibrations of microcantilevers in pure gases or air (mixture) at atmospheric pressure.

In the following section, the aim is to present some experimental data to demonstrate that this resonant frequency shift can be measured in the case of binary mixtures of varying concentrations.

\section{Measurement of THE FreQuenCy ShifT}

To verify if equation 9 can be used for gas detection, measurements have been made for two binary gas mixtures: helium in nitrogen and carbon dioxide in nitrogen in order to have one case with a smaller mass density than pure nitrogen and the other with a higher mass density.

\section{A. Microcantilevers, Actuation, Vibration Measurements}

The microcantilevers have been fabricated by ESIEE group (Paris, France, www.esiee.fr) using silicon technologies (Fig. 1).

The excitation of the microcantilever is made by electromagnetic actuation. The excitation arises due to the interaction between a magnetic field created by a magnet placed close to the chip and an electrical current passing through a conducting strip placed on the microcantilever. The Laplace electromagnetic force induces the structural motion. In order to detect the microcantilever oscillation, semiconductor strain gauges (boron-doped piezoresistors) are fabricated during the process. They are arranged in a half Wheatstone bridge configuration: a first gauge is located where the strains are maximum (the clamped-end of the beam) and the other is on the rigid substrate (Fig. 1). 
Preprint - Sensors and Actuators B, 143, 2010, pp. 555-560. (doi: 10.1016/j.snb.2009.09.062).

(a)

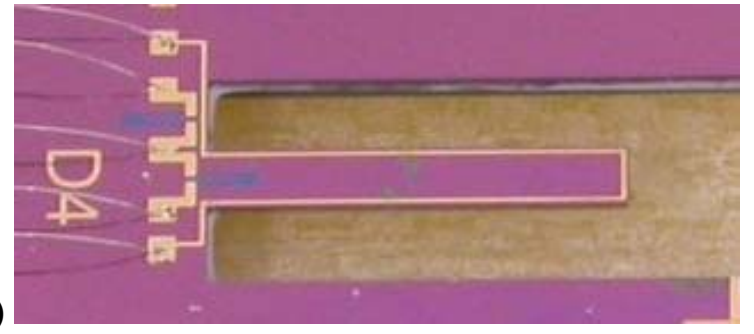

(b)

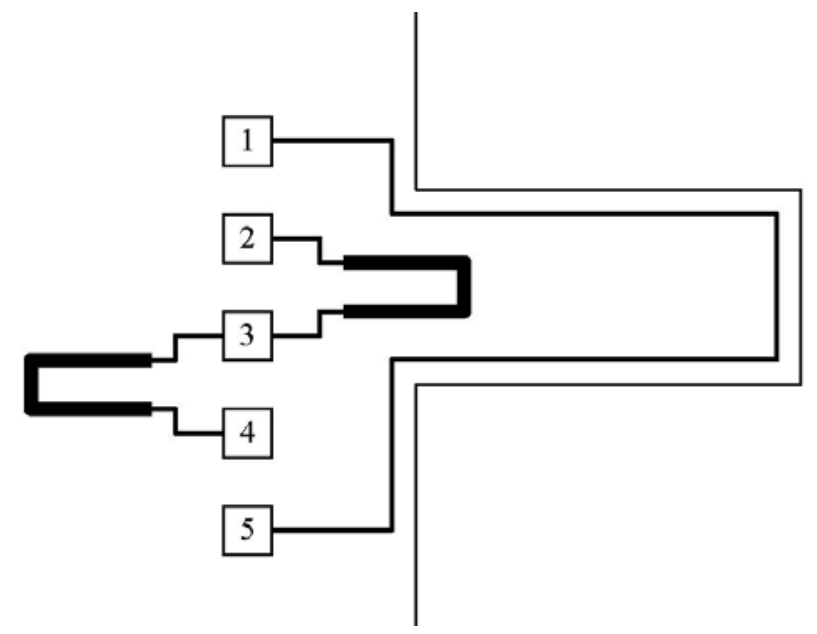

Figure 1. Silicon microcantilever with electromagnetic actuation and piezoresistive measurement: (a) photograph.

(b) electrical connection diagram: connection plots 2-3-4 represent piezoresistors and connection plots 1-5 denote the conducting path for electromagnetic actuation

\section{B. Experimental Setup}

The way to measure the resonant frequency shift is to actuate the microcantilever at a fixed frequency close to the resonant frequency, and to read out the piezoresistive signal phase. When the resonant frequency shifts, the phase of the microcantilever signal is modified. Near the resonant frequency the phase is almost a linear function of the frequency; thus, the measurement of the phase shift at a fixed frequency allows one to determine the resonant frequency shift.

The microcantilevers are placed in a gas chamber (total volume: $500 \mu \mathrm{l})$ under a controlled gas flow (100 to $1000 \mathrm{~cm}^{3} / \mathrm{min}$ ). Gas streams containing binary mixtures of the desired species (helium or carbon dioxide) and nitrogen are fed to the chamber using bottles of gas and a set of mass-flow controllers. 
Preprint - Sensors and Actuators B, 143, 2010, pp. 555-560. (doi: 10.1016/j.snb.2009.09.062).

\section{Measurements for a Helium/Nitrogen Mixture}

Fig. 2 shows examples of resonant frequency shift measurements for various helium concentrations in nitrogen $(2 \%, 1.5 \%, 1 \%$ and $0.5 \%)$ in the case of two microcantilever geometries among the five geometries studied. Based on the gas concentrations in the gas bottles ( $5 \%$ of helium in nitrogen and pure nitrogen) and the characteristics of the mass flow controllers, the flow rate required to generate the desired concentration mixture (a few percent) is $100 \mathrm{~cm}^{3} / \mathrm{min}$.

(a)

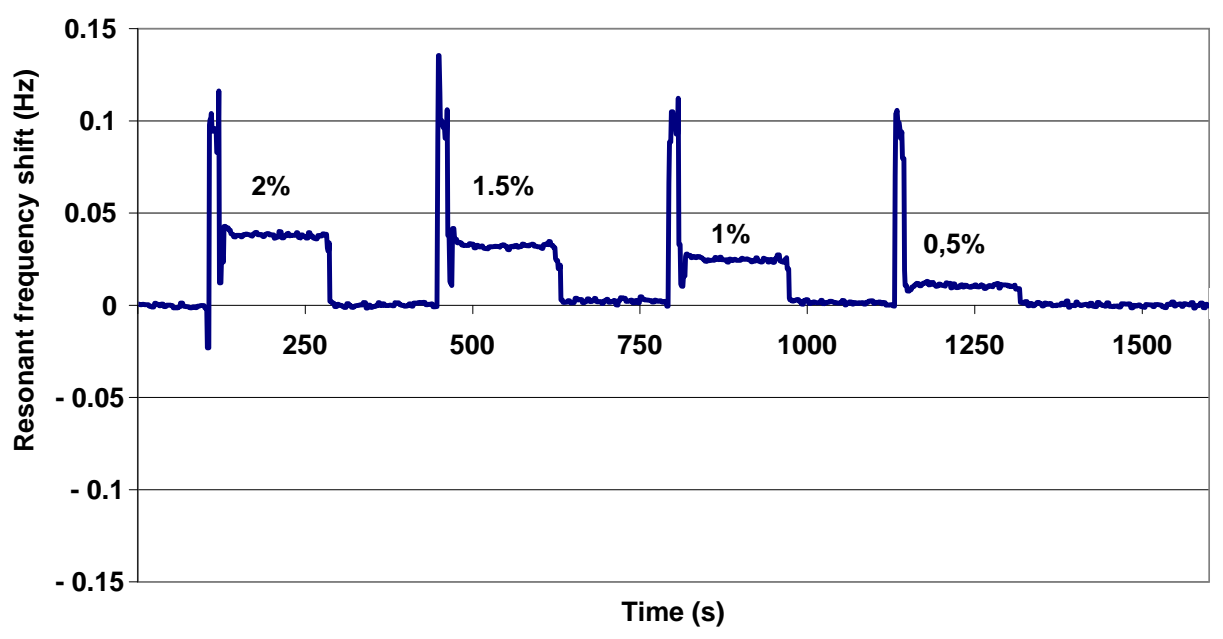

(b)

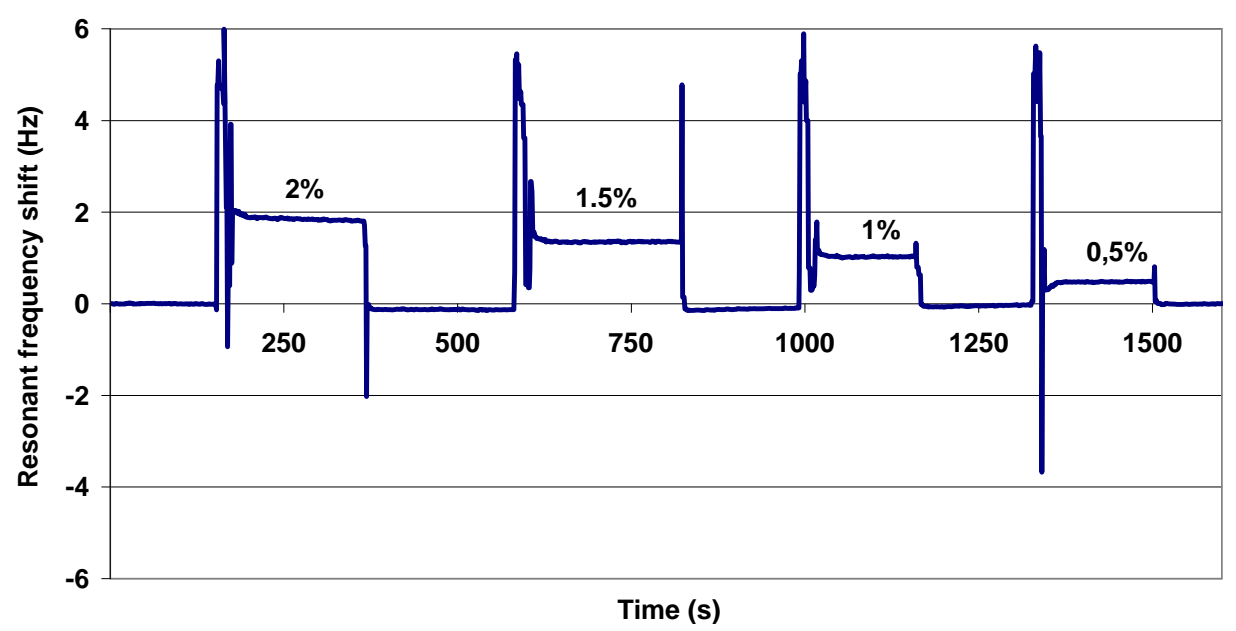

Figure 2. Example of resonant frequency shift measurement (drift removed) for different helium concentrations in nitrogen $(2 \%, 1.5 \%, 1 \%$ and $0.5 \%)$ for two different microcantilever geometries: (a) $L=6 \mathrm{~mm}, b=200 \mu \mathrm{m}$,

$$
h=109 \mu \mathrm{m} \text { (b) } L=1 \mathrm{~mm}, b=600 \mu \mathrm{m}, h=19 \mu \mathrm{m}
$$


Preprint - Sensors and Actuators B, 143, 2010, pp. 555-560. (doi: 10.1016/j.snb.2009.09.062).

These measurements show that in the presence of helium, the resonant frequency increases. This phenomenon is almost linear with the helium concentration and is reversible. Overshoots can be observed at each gas concentration, because of the mass flow controller command. The lower concentration step (0.5\%) gives a resonant frequency shift much higher than the frequency noise. This means that these types of microcantilevers could measure much smaller helium concentrations (limit of detection will be discussed in section IV). The amplitude of the resonant frequency shift depends on the microcantilever geometrical parameters.

\section{Measurements for a Carbon Dioxyde/Nitrogen Mixture}

The same experiments have been carried out with various carbon dioxide concentrations in nitrogen (1\%, $2 \%$, $3 \%, 4 \%$ ) with a $1000 \mathrm{~cm}^{3} / \mathrm{min}$ gas flow (this higher gas flow is due to the fact that in this case both gas bottles are with pure gases). Examples of resonant frequency shift measurements are presented in Fig. 3 for two different microcantilever geometries. 
Preprint - Sensors and Actuators B, 143, 2010, pp. 555-560. (doi: 10.1016/j.snb.2009.09.062).

(a)
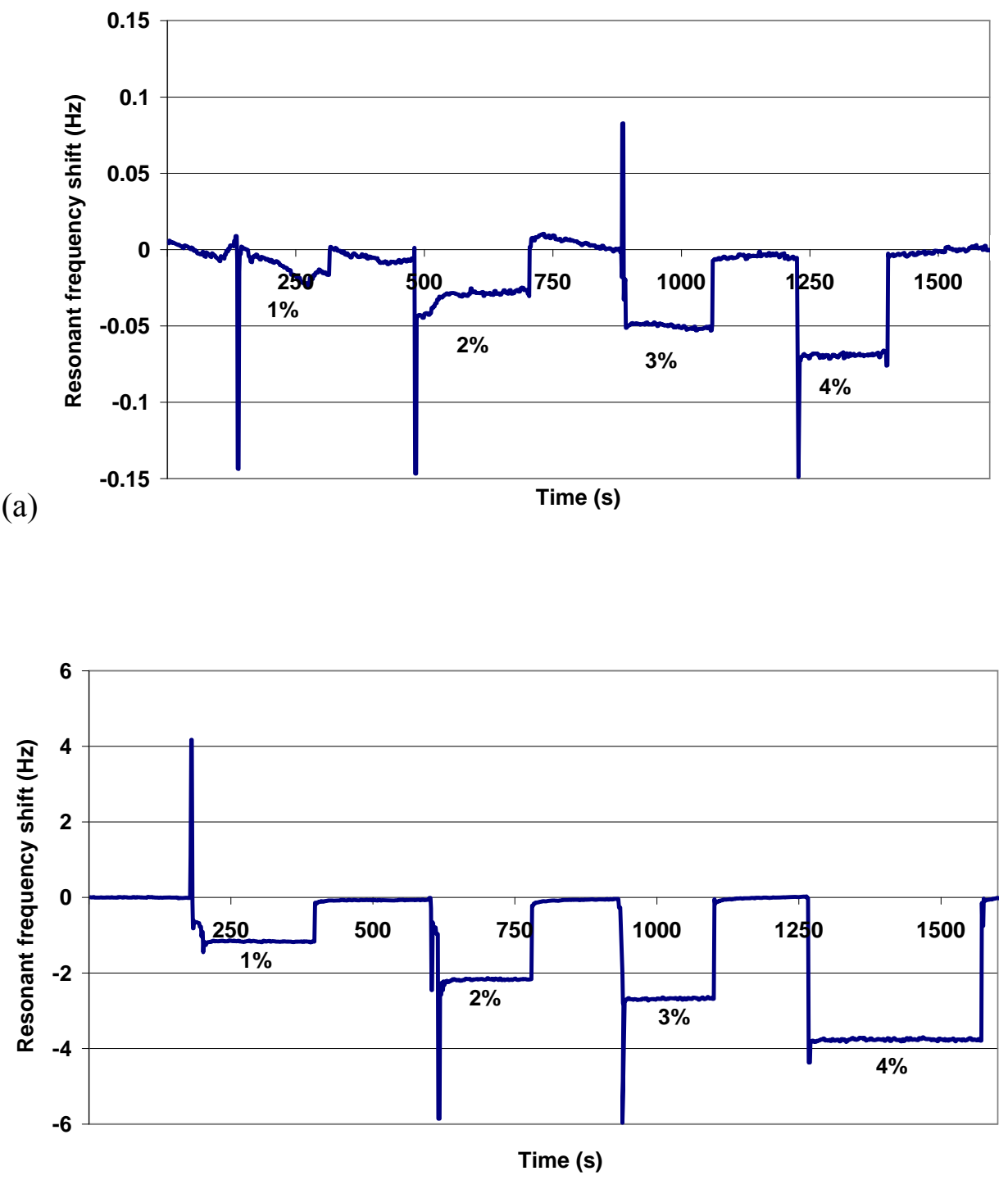

Figure 3. Example of resonant frequency shift measurement (drift removed) for different carbon dioxide concentrations in nitrogen $(1 \%, 2 \%, 3 \%$ and $4 \%)$ for two different microcantilever geometries:. (a) $L=6 \mathrm{~mm}$, $b=200 \mu \mathrm{m}, h=109 \mu \mathrm{m}$ (b) $L=1 \mathrm{~mm}, b=600 \mu \mathrm{m}, h=19 \mu \mathrm{m}$

A negative resonant frequency shift is observed due to the carbon dioxide concentrations. As with the experiments in the helium/nitrogen mixtures, the sensitivity depends on the microcantilever geometry, the 
Preprint - Sensors and Actuators B, 143, 2010, pp. 555-560. (doi: 10.1016/j.snb.2009.09.062).

measurements exhibit reversibility, and the smallest output signal in the experiments is much higher than the noise. In addition, quasi-linearity is observed.

\section{Discussion}

The aim of this section is to compare the expected resonant frequency shifts (based on equation 9 of section II,) to the experimentally measured shifts presented in section III.

\section{A. Density and Viscosity of a Binary Mixture}

First, in order to apply the theoretical equation it is necessary to know the mass density and viscosity of a binary mixture.

Using the definition of the mass density, in the case of a binary mixture it can be expressed as a function of the mass densities of each component:

$$
\rho_{f}=x_{1} \rho_{1}+\left(1-x_{1}\right) \rho_{2}
$$

with $\rho_{1}$ and $\rho_{2}$ the mass densities of gas 1 and gas 2, respectively, and $x_{1}$ the volume fraction of gas 1 .

Contrary to the case of mass density, the viscosity of a binary mixture is not easy to obtain using the viscosity definition. An approximate expression for the viscosity of gas mixtures was derived by Sutherland in 1895. It is based on a simple mean-free-path model and, for a binary gas mixture, is given by [11]

$$
\eta=\frac{x_{1} \eta_{1}}{x_{1}+\left(1-x_{1}\right) \Phi_{12}}+\frac{\left(1-x_{1}\right) \eta_{2}}{1-x_{1}+x_{1} \Phi_{21}}
$$

with $\eta_{1}$ and $\eta_{2}$ being the viscosities of gas 1 and gas 2 , respectively.

Concerning the expressions for $\Phi_{12}$ and $\Phi_{21}$, many attempts to predict these parameters have been made. Wilkes [12] derived the following expressions:

$$
\Phi_{12}=\left[8\left(1+\frac{M_{1}}{M_{2}}\right)\right]^{-1 / 2}\left[1+\left(\frac{\eta_{1}}{\eta_{2}}\right)^{1 / 2}\left(\frac{M_{2}}{M_{1}}\right)^{1 / 4}\right]^{2}
$$


Preprint - Sensors and Actuators B, 143, 2010, pp. 555-560. (doi: 10.1016/j.snb.2009.09.062).

$$
\Phi_{21}=\left[8\left(1+\frac{M_{2}}{M_{1}}\right)\right]^{-1 / 2}\left[1+\left(\frac{\eta_{2}}{\eta_{1}}\right)^{1 / 2}\left(\frac{M_{1}}{M_{2}}\right)^{1 / 4}\right]^{2}
$$

where $M_{1}$ and $M_{2}$ are the molar masses of the gases.

The average deviation between calculated and experimental data for thirteen mixtures involving different gases (CO2, O2, CO, H2, CH4, N2, etc.) is 1.9\% [12]. Brokwak [13] gave other more complicated expressions resulting in almost the same numerical results: for 280 mixtures involving 25 gas pairs (including He/N2 and $\mathrm{CO} 2 / \mathrm{N} 2$ ), the average error when comparing with experimental data is $0.7 \%$ [13]. Both of these methods use only the viscosity and molecular mass of the two pure components.

\section{B. Case of $\mathrm{He} / \mathrm{N}_{2}$ and $\mathrm{CO}_{2} / \mathrm{N}_{2}$ Mixtures}

Considering that helium, carbon dioxide and nitrogen are perfect gases, their mass densities can be calculated for a temperature of $25^{\circ} \mathrm{C}$ and a pressure of $1000 \mathrm{hPa}$. For these temperature and pressure conditions, the mass density of nitrogen, helium and carbon dioxide are, respectively, $\rho_{\mathrm{N}_{2}}=1.13 \mathrm{~kg} / \mathrm{m}^{3}, \rho_{\mathrm{He}}=0.161 \mathrm{~kg} / \mathrm{m}^{3}$, $\rho_{\mathrm{CO}_{2}}=1.77 \mathrm{~kg} / \mathrm{m}^{3}$.

The viscosities of the gases also depend on the temperature:

$$
\eta=2.669310^{-6} \frac{\sqrt{M T}}{\sigma^{2} \Omega^{(2,2)^{*}}}
$$

with $M$ the molar mass $\left(g \cdot \mathrm{mol}^{-1}\right), T$ the temperature $(K), \sigma$ a length for the expression of the interaction potential $(\AA)$ and $\Omega^{(2,2)^{*}}$ the double normalized collision integral for the Lennard-Jones potential:

$$
\Omega^{(2,2)^{*}}=1.1614\left(T^{*}\right)^{-0.1487}+0.5249 e^{-0.7732 T^{*}}+2.1618 e^{-2.4378 T^{*}}
$$

with the normalized temperature $T^{*}$ defined by

$$
T^{*}=\frac{k T}{\varepsilon}
$$


Preprint - Sensors and Actuators B, 143, 2010, pp. 555-560. (doi: 10.1016/j.snb.2009.09.062).

where $k=1.38066210^{-23} \mathrm{JK}^{-1}$ is the Boltzmann constant and $\varepsilon$ the energy in the action potential $(J)$.

Using equations 14-16 and the numerical values of the different parameters for each gas, the viscosities at $25^{\circ} \mathrm{C}$ for nitrogen, helium and carbon dioxide are, respectively, $\eta_{N_{2}}=17.8 \mu$ Pa.s, $\eta_{H_{e}}=19.9 \mu$ Pa.s, $\eta_{\mathrm{CO}_{2}}=14.8 \mu$ Pa.s.

Using equations 10-11, it is possible to calculate the mass density and viscosity of the binary mixtures for different concentrations of carbon dioxide or helium. The results of the relative variations are summarized in Table I.

TABLE I. RELATIVE VARIATION OF DENSITY AND VisCOSITY

\begin{tabular}{|c|c|c|c|c|}
\hline \multirow{2}{*}{$\begin{array}{c}\text { He } \\
\text { or } \mathbf{C O}_{2}\end{array}$} & $\frac{2}{|c|} \mathbf{H e} / \mathbf{N}_{2}$ Mixture & \multicolumn{2}{c|}{$\mathbf{C O}_{2} / \mathbf{N}_{2}$ Mixture } \\
\cline { 2 - 5 } & $\rho_{f}$ & $\frac{d \eta}{\eta}$ & $\frac{d \rho_{f}}{\rho_{f}}$ & $\frac{d \eta}{\eta}$ \\
\hline $0.1 \%$ & $-0.0857 \%$ & $0.0144 \%$ & $0.0571 \%$ & $-0.0222 \%$ \\
\hline $1 \%$ & $-0.857 \%$ & $0.144 \%$ & $0.571 \%$ & $-0.222 \%$ \\
\hline
\end{tabular}

\section{Experiment/Modeling Comparison}

In the modeling of the resonant frequency shift (equation 9) there are two terms: one due to the mass density variation and the other due to the viscosity variation. In order to understand the relative influence of the two effects, both terms have been calculated for various microcantilever geometries (Table II). The numerical results are presented in Table III for $1 \%$ concentration of helium or carbon dioxide.

TABLE II. MICROCANTILEVER GEOMETRIES

\begin{tabular}{|c|c|c|c|}
\hline beam & $\begin{array}{c}\text { Length } \boldsymbol{L} \\
\mathbf{( \mu m )}\end{array}$ & $\begin{array}{c}\text { Width } \boldsymbol{b} \\
(\boldsymbol{\mu m})\end{array}$ & $\begin{array}{c}\text { Thickness } \boldsymbol{h} \\
(\boldsymbol{\mu m})\end{array}$ \\
\hline $\mathbf{1}$ & 6000 & 200 & 109 \\
\hline $\mathbf{2}$ & 2000 & 200 & 57 \\
\hline
\end{tabular}


Preprint - Sensors and Actuators B, 143, 2010, pp. 555-560. (doi: 10.1016/j.snb.2009.09.062).

\begin{tabular}{|c|c|c|c|}
\hline beam & $\begin{array}{c}\text { Length } \boldsymbol{L} \\
\mathbf{( \mu m )}\end{array}$ & $\begin{array}{c}\text { Width } \boldsymbol{b} \\
\mathbf{( \mu m )}\end{array}$ & $\begin{array}{c}\text { Thickness } \boldsymbol{h} \\
(\boldsymbol{\mu m})\end{array}$ \\
\hline $\mathbf{3}$ & 2000 & 1000 & 112 \\
\hline $\mathbf{4}$ & 6000 & 1000 & 115 \\
\hline $\mathbf{5}$ & 1000 & 600 & 19 \\
\hline
\end{tabular}

TABLE III. EFFECTS ON THE FREQUENCY SHIFT OF THE DENSITY AND VISCOSITY VARIATIONS (1\% GAS CONCENTRATION)

\begin{tabular}{|c|c|c|c|c|}
\hline \multirow{2}{*}{ beam } & \multicolumn{2}{|c|}{ He/ $\mathbf{N}_{\mathbf{2}}$ Mixture } & \multicolumn{2}{c|}{$\mathbf{C O}_{2} / \mathbf{N}_{\mathbf{2}}$ Mixture } \\
\cline { 2 - 5 } & $a_{1} b \frac{d \rho_{f}}{\rho_{f}}$ & $\frac{a_{2} \delta}{2} \frac{d \eta}{\eta}$ & $a_{1} b \frac{d \rho_{f}}{\rho_{f}}$ & $\frac{a_{2} \delta}{2} \frac{d \eta}{\eta}$ \\
& $(\mu \mathrm{m})$ & $(\mu \mathrm{m})$ & $(\mu \mathrm{m})$ & $(\mu \mathrm{m})$ \\
\hline $\mathbf{1}$ & -1.81 & 0.0952 & 1.21 & -0.146 \\
\hline $\mathbf{2}$ & -1.81 & 0.0439 & 1.21 & -0.0672 \\
\hline $\mathbf{3}$ & -9.04 & 0.0313 & 6.03 & -0.0479 \\
\hline $\mathbf{4}$ & $-9.04^{6}$ & 0.0927 & 6.03 & -0.142 \\
\hline $\mathbf{5}$ & -5.43 & 0.0380 & 3.62 & -0.0582 \\
\hline
\end{tabular}

The numerical results presented in Table III show that, for the considered geometries, the term due to viscosity variation is negligible compared to that due to mass density variation. (The ratio of the terms is between 10 and 300). As a result, equation 9 can be simplified for the present application to

$$
\frac{d f_{r}}{f_{r}}=-\frac{\pi a_{1}}{8} \frac{b}{h} \frac{d \rho_{f}}{\rho}
$$

As expected from the modeling, the measured resonant frequency (Figs. 2-3) increases in the case of helium because of the decreasing mass density of the surrounding medium, whereas it decreases in the case of carbon dioxide because of increasing mass density. 
Preprint - Sensors and Actuators B, 143, 2010, pp. 555-560. (doi: 10.1016/j.snb.2009.09.062).

In order to accurately compare equation 17 with measurements made with the five studied microcantilever geometries (Table II), linear regression has been performed on the experimental data to obtain numerical values of the slope of the relative resonant frequency shift versus the mass density of the gas (Fig. 4). The comparison of the slopes based on modeling and measurement is shown in Fig. 5.

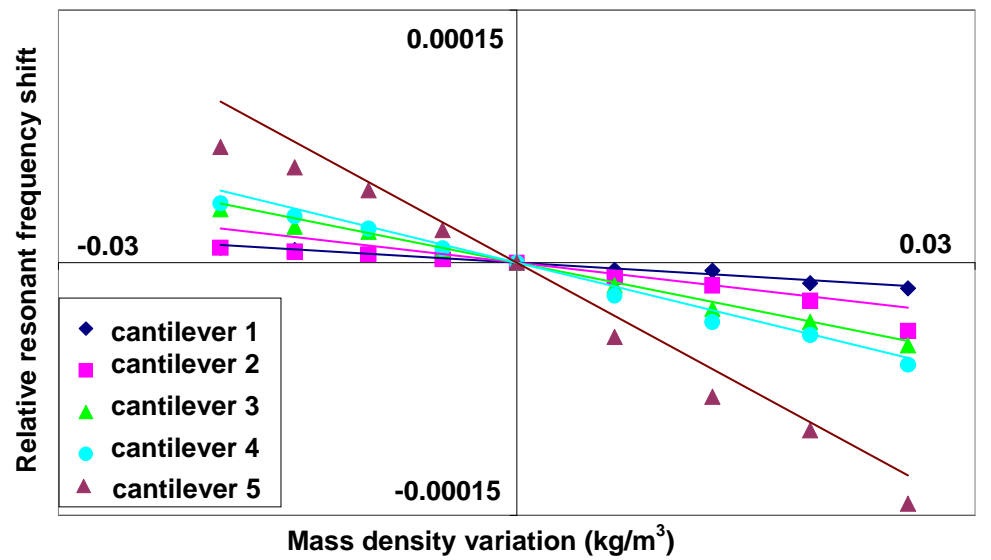

Figure 4. Relative resonant frequency shifts for the five microcantilever geometries in the cases of $\mathrm{He} / \mathrm{N}_{2}$ and $\mathrm{CO}_{2} / \mathrm{Ne}$ mixtures of different concentrations. Linear regression has been made on the experiemental data (discrete markers)

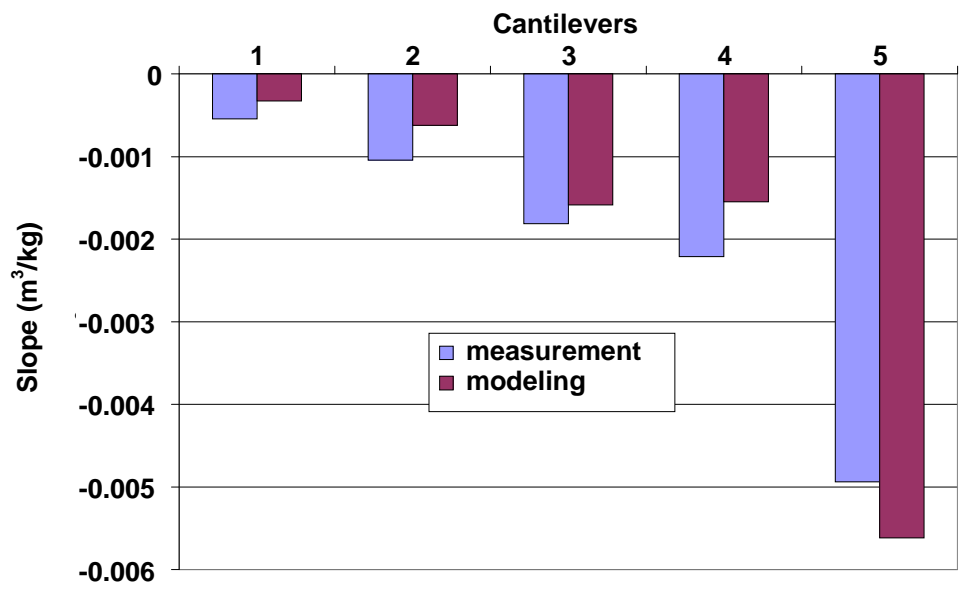

Figure 5. Comparison of the slope $\left(\mathrm{m}^{3} / \mathrm{kg}\right.$ ) obtained by modeling (eq. 17) and measurement for the five microcantilevers. 
Preprint - Sensors and Actuators B, 143, 2010, pp. 555-560. (doi: 10.1016/j.snb.2009.09.062).

A very good agreement is observed between measurements and analytical modeling for the various microcantilever geometries and gas concentrations (Fig.5).

\section{Limit of Detection}

The validity of equation 17 has been demonstrated, which means that this equation can be used to determine optimal values of the microcantilever geometric parameters and the associated performance of such microsensors.

For a given gas mixture, the relative resonant frequency shift increases with the width-to-thickness ratio of the microcantilever. Wide, thin microcantilevers will thus be preferred. This dimensional choice is confirmed by Fig. 4: the slope increases with the ratio $b / h$. For the five tested microcantilevers the most sensitive is $\# 5$ and the least sensitive is \#1.

For the tested microcantilevers the resonant frequency is between $4 \mathrm{kHz}$ and $50 \mathrm{kHz}$. Considering the experimental limit of frequency measurement of $10 \mathrm{mHz}$, it is possible using equations 10 and 17 to estimate the limit of detection in both cases of helium/nitrogen and carbon dioxide/nitrogen mixtures (Table IV).

TABLE IV. LIMIT OF DETECTION

\begin{tabular}{|c|c|c|}
\hline beam & $\begin{array}{c}\text { He limit of detection } \\
\mathbf{m g} / \mathbf{m}^{\mathbf{3}} \mathbf{( \mathbf { p p m } )}\end{array}$ & $\begin{array}{c}\mathbf{C O}_{\mathbf{2}} \text { limit of } \\
\text { detection } \\
\mathbf{m g} / \mathbf{m}^{\mathbf{3}} \mathbf{( \mathbf { p p m } )}\end{array}$ \\
\hline $\mathbf{1}$ & $1210(7562)$ & $20287(11461)$ \\
\hline $\mathbf{2}$ & $134(840)$ & $2254(1273)$ \\
\hline $\mathbf{3}$ & $27(168)$ & $451(255)$ \\
\hline $\mathbf{4}$ & $242(1512)$ & $4057(2292)$ \\
\hline $\mathbf{5}$ & $11(70)$ & $188(106)$ \\
\hline
\end{tabular}

\section{CONCLUSION}

Compared to classical resonant microcantilever-based chemical sensors with sensitive coating, the uncoated microsensors studies herein have smaller response times because there is no diffusion time in the response. The 
Preprint - Sensors and Actuators B, 143, 2010, pp. 555-560. (doi: 10.1016/j.snb.2009.09.062).

lack of the sensitive coating leads to a more reliable and reversible behavior because there is no significant absorption and desorption phenomenon. The results of this study demonstrate that uncoated microsensors may serve as viable devices for detection of specific concentrations of one gas in a binary mixture. The sensitivity and resolution of such sensors will be larger for those cases in which the difference between the mass densities of the two gases is higher.

\section{ACKNOWLEDGMENT}

This work has been supported by the EADS Foundation.

\section{REFERENCES}

[1] M. Sepaniak, P. Datskos, N. Lavrik and C. Tipple, "Microcantilever transducers: a new approach in sensor technology," Analytical Chemistry, A-Pages, 74 (21), pp. 568A-575A, 2002.

[2] K.M. Goeders, J.S. Colton, L.A. Bottomley, "Microcantilevers: sensing chemical interactions via mechanical motion," Chemical Reviews, vol. 108, pp.522-542, 2008.

[3] F.R. Blom, S. Bouwstra, M. Elwenspoek and J.H.J. Fluitman, Dependance of the quality factor of micromachined silicon beam resonators on pressure and geometry, Journal of vacuum science and technology, B, 10, nº1 (1992) pp. 19-26.

[4] H. Hosaka, K. Itao and S. Kuroda, Damping characteristics of beam-shaped micro-oscillators, Sensors and Actuators A, 49 (1995) pp. 87-95.

[5] E. Sader, Frequency response of cantilever beams immersed in viscous fluids with applications to the atomic force microscope, Journal of Applied Physics, 84 (1998) pp. 64-76.

[6] R.D. Blevins, Formulas for natural frequency and mode shape, Van Nostrand Reinhold Company, 1979.

[7] K.Y. Yasumura, T.D. Stowe, E.M. Chow, T. Pfafman, T.W. Kenny, B.C. Stipe and D. Rugar, Quality factors in micron- and submicron-thick cantilevers, Journal of microelectromechanical systems, 9, (2000) pp. 117-125.

[8] F. Lochon, I. Dufour, D. Rebière, "A microcantilever chemical sensors optimization by taking into account losses", Sensors and Actuators B, Vol 118, (2006), pp. 292-296.

[9] A. Maali, C. Hurth, R. Boisgard, C. Jai, T. Cohen-Bouhacina and J.P. Aimé, Hydrodynamics of oscillating atomic force microscopy cantilevers in viscous fluids, Journal of Applied Physics, 97 (2005) 074907. 
Preprint - Sensors and Actuators B, 143, 2010, pp. 555-560. (doi: 10.1016/j.snb.2009.09.062).

[10] S. Boskovic, J.W.M. Chon, P. Mulvaney and J.E. Sader, Rheological measurements using microcantilevers, Journal of Rheology, 46 (2002) pp. 891-899.

[11] W. Sutherland, The viscosity of mixed gases, Philosophical Magazine and Journal of Science, 40, pp. 421431 (1895)

[12] C.R Wilke, A viscosity equation for gas mistures, Journal of Chemical physics, 18, pp. 517-518 (1950).

[13] P.S. Brokaw, Predicting transport properties of dilute gases, Industrial Engineering Chemistry Process Design and Development, 8, pp. 240-253 (1968). 